\title{
Small cytoplasmic RNA of Bacillus brevis: transcriptional and phylogenetic analysis
}

\author{
Kouji Nakamura, Mitsuru Nishiguchi, Maki Minemura, Kiyofumi Honda \\ and Kunio Yamane
}

Author for correspondence: Kouji Nakamura. Tel: +8129853 6419. Fax: +81298536006.

Institute of Biological Sciences, University of Tsukuba, Tsukuba-shi, Ibaraki 305, Japan

\begin{abstract}
Using a DNA fragment of Bacillus subtilis SCRNA as a probe, a Bacillus brevis gene encoding the small cytoplasmic RNA was cloned and characterized. $B$. brevis SCRNA consists of $\mathbf{2 7 3}$ nucleotides; the sequence has comparatively low homology (approximately $70 \%$ ) with other Bacillus sequences. Phylogenetic analysis indicated that $B$. brevis forms a line of descent distinct from other Bacillus species. However, despite the low overall homology, both functional nucleotide sequence and secondary structural features defined among signal recognition particle (SRP) RNA family members were well conserved.
\end{abstract}

Keywords: small cytoplasmic RNA, signal recognition particle, Bacillus brevis, phylogenetic analysis

\section{INTRODUCTION}

Signal recognition particle (SRP) is a cytoplasmic ribonucleoprotein which targets the ribosomes translating nascent secretory proteins to the endoplasmic reticulum membrane (Walter \& Lingappa, 1986). The extensively studied canine particle is composed of one RNA molecule (here referred to as SRP RNA) and six polypeptides (Walter \& Blobel, 1980, 1982). SRP RNA-like RNAs have been identified in a wide range of eukaryotes, including humans, plants and yeast, and in a wide variety of archaeobacteria (Larsen \& Zwieb, 1991).

The small cytoplasmic RNA (scRNA; 271 nucleotides) of Bacillus subtilis is considered to be a member of the SRP RNA family. Although dissimilar in size and overall primary structure to mammalian SRP RNA, it contains domains of which the secondary structures correspond to domains I, II and IV of the SRP RNA-like RNAs of archaeobacteria and eukaryotes. The nucleotide sequence in domain IV is highly conserved (Poritz et al., 1988; Kaine, 1990). Functional analyses have shown that this RNA is essential for the cell viability of $B$. subtilis (Nakamura et al., 1992a). To identify the functional domains of scRNA, knowledge of its structure is required, and phylogenetic comparisons between homologous RNAs have proved useful in elucidating a higher-order

\footnotetext{
Abbreviations: scRNA, small cytoplasmic RNA; SRP, signal recognition particle.

The GenBank accession number for the nucleotide sequence reported in this paper is D14472.
}

RNA structure (Romero \& Blackburn, 1991; Haas et al., 1991). It is therefore useful to compare scRNAs from more closely related species. The scRNA cataloguing studies have so far been restricted to two Bacillus species, B. subtilis and B. stearothermophilus (Struck \& Erdmann, 1990). Consequently, it has not been possible to draw reliable conclusions about the phylogenetic structure of the genus. To identify the conserved nucleotides and structural elements of scRNA, we determined the nucleotide sequence of scRNA from various bacilli (Nakamura $e t$ al., 1992b). DNA fragments corresponding to the mature scRNA of ten Bacillus species were obtained by polynucleotide chain reaction (PCR), using primers based upon comparisons of the nucleotide sequence of scRNAs between $B$. subtilis and B. stearothermophilus. A positively amplified DNA fragment was not obtained in B. brevis.

In this report, we describe the cloning and nucleotide sequencing of the entire structural portion of the B. brevis scRNA gene. We also determined the $5^{\prime}$ and $3^{\prime}$ ends of mature scRNA. A pairwise comparison of scRNA sequences from 13 Bacillus species indicates that B. brevis forms a distinct line of descent in the genus Bacillus.

\section{METHODS}

Bacterial strains. Escherichia coli JM109 (Yanisch-Perron et al., 1985) and NM514 [bsdR $514\left(\mathrm{r}_{\mathrm{k}}^{-} \mathrm{m}_{\mathrm{k}}^{-}\right) \operatorname{argH}$ galE galX str $A$ lycB7 $\left(H f^{+}\right)$] were used as cloning hosts. B. brevis ATCC 5246 was obtained from the Bacillus Genetic Stock Center (Ohio State University). The cells were cultured in $\mathrm{L}$ broth at $37^{\circ} \mathrm{C}$.

DNA and RNA preparation. Chromosomal DNA of B. brevis ATCC 5246 was prepared by the method of Saito \& Miura 


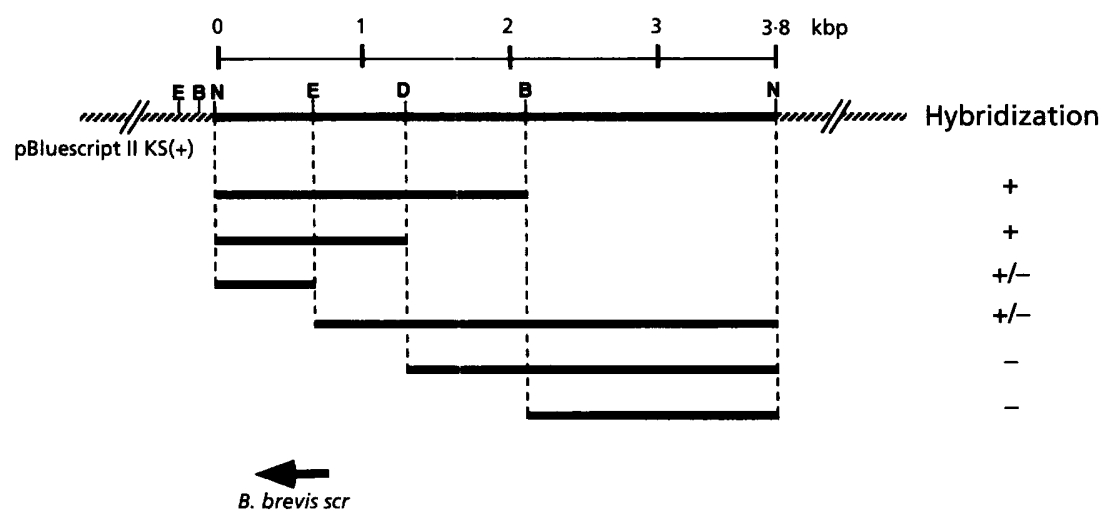

Fig. 1. Physical map and location of the SCRNA coding region (scr) in the subcloned $3.8 \mathrm{~kb}$ Notl fragment of pTUE825. The arrow indicates the direction of transcription of the scr. B, BamHI site; E, EcoRI site; N, Notl site. The location of the scRNA coding region was determined by Southern hybridization, using the $B$. subtilis scRNA gene as the probe. ,$++I-$ and - indicate the strength of hybridization signals.

(1963) and stored at $4{ }^{\circ} \mathrm{C}$ in TE buffer. Total RNA was isolated from $B$. brevis ATCC 5246 grown to the mid-exponential phase $\left(\mathrm{OD}_{600}, 0.5-0.6\right)$ as described previously (Nakamura et al., 1992a) and stored at $-80^{\circ} \mathrm{C}$ in distilled water treated with diethyl pyrocarbonate (DEPC).

DNA Southern hybridization. DNA preparations digested with restriction enzymes were separated by agarose gel electrophoresis and transferred onto Gene Screen Plus nylon membranes (NEN Research Products). Hybridization under low stringency proceeded as described previously (Nakamura et al., 1990). The 271 bp DNA fragment, corresponding to mature scRNA, was amplified from the chromosomal DNA of $B$. subtilis 168 using PCR with the synthetic oligonucleotide primers 5' T'T'TGCCGTGCTAAGCGGG $3^{\prime}$ and $5^{\prime}$ TACCGTGCACCT'TCTGTC 3'. The amplified DNA fragment was inserted at the SmaI site of M13mp10. To prepare the specific probe which cannot hybridize to the 4.5S RNA gene of $E$. coli but can hybridize to Bacillus scRNA, 42 nucleotides in domain IV, corresponding to +144 to +185 , were deleted by oligonucleotide-directed mutagenesis, using the mutagenic oligonucleotide 5' CGGCACATGAGAGGTAAT'TCCCATTGCGCA 3'. After KpnI-BamHI digestion of the doublestranded M13 phage DNA, the 234-nucleotide fragment of Bacillus subtilis scRNA gene without domain IV was isolated by polyacrylamide gel electrophoresis. This fragment was labelled with $\left[\alpha-{ }^{32} \mathrm{P}\right] \mathrm{dCTP}$ using a random primer DNA labelling kit (Takara Shuzo Co.) and used as the probe.

DNA sequence analysis. DNA was sequenced by dideoxy chain termination (Sanger et al., 1977). Both strands were entirely sequenced, and all ends of the fragments overlapped.

Primer extension. The oligonucleotide (NK1; 5' AGGATAGGAATTCACACCCTGGCTATT $3^{\prime}$ ) used in primer extension was synthesized automatically (Applied Biosystems). Total B. brevis RNA $(40 \mu \mathrm{g})$ and $5 \times 10^{4}$ c.p.m. of the ${ }^{32} \mathrm{P}$-labelled oligonucleotide primer were hybridized at $40^{\circ} \mathrm{C}$ overnight. RAV-2 (Rous associated virus 2) reverse transcriptase was added and the mixture was incubated at $42^{\circ} \mathrm{C}$ for $1 \mathrm{~h}$. The reaction products were analysed on DNA sequencing gels. The length of extended DNA fragment was estimated by comparison with sequencing ladders generated from M13mp10.

RNAse protection assay. A BamHI-EcoRI fragment, including a $350 \mathrm{bp} 3^{\prime}$ portion of the B. brevis scRNA gene, was inserted between the BamHI and EcoRI sites of Bluescript II SK(-) under the control of the bacteriophage T3 promoter. T3 RNA polymerase and $\left[\alpha^{32} \mathrm{P}\right]$ CTP were used to synthesize the labelled probe, which encompasses the $3^{\prime}$ portion of $B$. brevis scRNA, and to which it is complementary. The RNase protection assay was performed as described previously (Nakamura et al., 1992a), using total RNA $(10 \mu \mathrm{g})$ and $3.9 \times 10^{6}$ c.p.m. of the ${ }^{32}$ P-labelled probe.

\section{RESULTS AND DISCUSSION}

\section{Cloning and sequencing of the SCRNA gene region}

To verify the existence of the scr gene in chromosomal DNA of B. brevis ATCC 5246, Southern hybridization was performed using the $B$. subtilis scRNA gene without domain IV as a probe. A $3.8 \mathrm{~kb}$ PvuII-HincII fragment specifically hybridized with the probe. PvuII-HincIIdigested chromosomal DNA from B. brevis ATCC 5246 was resolved by agarose gel electrophoresis. Fragments of 3-5 $\mathrm{kb}$ were extracted from the gel and ligated with the EcoRI-NotI adaptor (5' dAAT'TCGCGGCCGCT $3^{\prime}$, $5^{\prime}$ dAGCGGCCGCG 3') to create EcoRI sites at both ends. The resultant DNA fragments were inserted into $\lambda$ gt10, packed in vitro, then transfected into $E$. coli NM514. Among 5000 plaques, 25 carried the $3.8 \mathrm{~kb}$ insert. Plasmid pTUE825 was constructed by subcloning the $3.8 \mathrm{~kb}$ Not I fragment from the phage DNA into the NotI site of pBluescript II $\mathrm{KS}(+)$. Physical mapping followed by Southern analysis of pTUE 825 revealed that a NotI-DraI fragment of about $1 \cdot 1 \mathrm{~kb}$ contains the scRNA coding region (Fig. 1) and the entire region of this fragment was sequenced (Fig. 2a). The sequences were aligned with the mature form of $B$. subtilis scRNA (Fig. 2b). The scRNA coding region of $B$. brevis deduced from this alignment, underlined in Fig. 2(a), is about $70 \%$ identical to that of $B$. subtilis scRNA. About $40 \mathrm{bp}$ upstream from the putative scRNA coding region, a possible promoter region was identified ( -35 box, TTGCTA; -10 box, TATTCT), which was highly homologous with the consensus sequence recognized by the B. subtilis $\sigma^{\mathrm{A}}$ RNA 
(a)

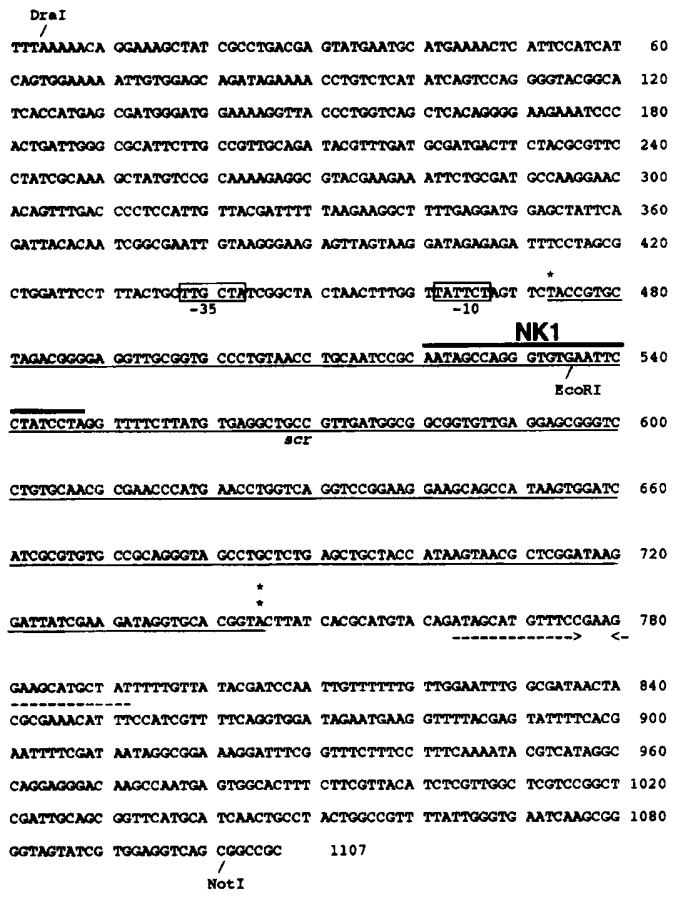

(b)

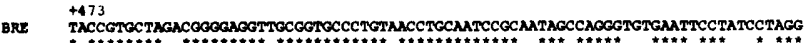
SUB TTTOCCGTOCTAMGCGGGGNGGTAGCGGTOCCCTGL-ACCTGCANTCCGCTCTAG-CAOGG-CCGAATCCCTTCTCGAGG

BRx TH51

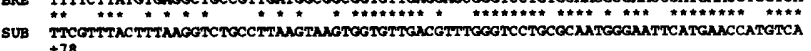

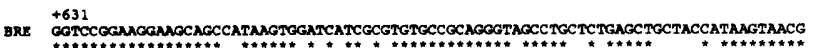

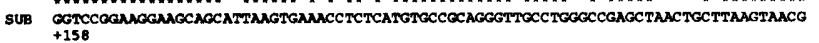



SUB CTMDOGTA-OCGMTCGACAGMAOTROCACOGTA

Fig. 2. (a) Nucleotide sequence of the chromosomal segment containing the $B$. brevis scRNA gene. The nucleotide sequence of the Dral-Notl fragment in pTUE825 was determined and numbered arbitrarily. The sequence of mature scRNA of $B$. brevis deduced by alignment with that of $B$. subtilis is underlined. The presumed promoter elements $(-10$ and -35$)$ are boxed. The positions of the $5^{\prime}$ and $3^{\prime}$ ends of the mature scRNA determined in Fig. 3 are indicated by single and double asterisks, respectively. Palindromic sequences which may serve as transcriptional terminator are indicated by converging arrows. A nucleotide sequence complementary to the synthetic oligonucleotide primer used in primer extension is overlined. (b) A comparison of the primary structure of $B$. subtilis SCRNA (SUB) and the nucleotide sequence determined here for $B$. brevis sCRNA (BRE). Asterisks indicate identical nucleotides between the two sequences.

polymerase ( -35 box, TTGACA ; -10 box, TATAAT). About 30 bp downstream from the scRNA coding region there is an almost perfect palindromic sequence comprising $26 \mathrm{bp} \quad\left(\Delta G=-25.7 \mathrm{kcal} \mathrm{mol}^{-1} ; \quad-107.5 \mathrm{~kJ}\right.$ $\mathrm{mol}^{-1}$ ) as calculated by the method of Tinoco et al. (1973), followed by a run of thymidine residues (Fig. 3c). This (a)

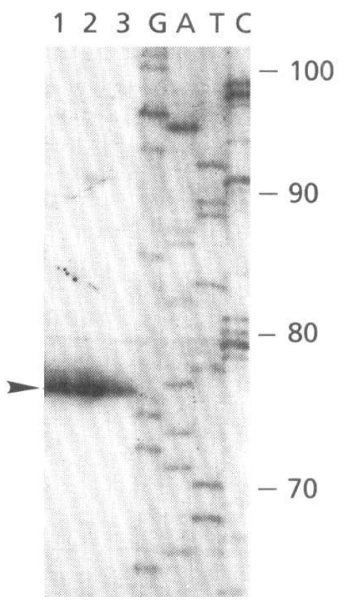

(c)

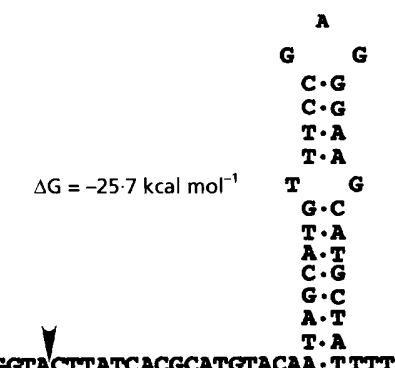

(b)

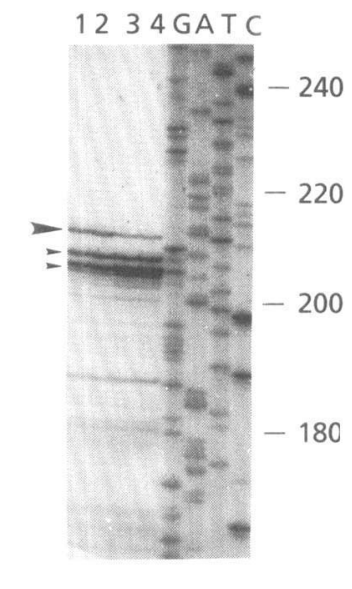

Fig. 3. Determination of the $5^{\prime}$ and $3^{\prime}$ ends of $B$. brevis scRNA. (a) Mapping the $5^{\prime}$ terminus by primer extension using primer NK1 (Fig. 2a). RNA samples were prepared from B. brevis cultured for 6 (lane 1), 9 (lane 2) and $12 \mathrm{~h}$ (lane 3) after inoculation. Sequencing reactions (G, $A, T$ and $C$ ) served as size markers. (b) Mapping the $3^{\prime}$ terminus by means of an RNase protection assay of the SCRNA gene transcript. RNA samples were prepared from $B$. brevis cultured for 3 (lane 1), 6 (lane 2), 9 (lane 3 ) and $12 \mathrm{~h}$ (lane 4). Sequencing reactions (G, A, T and C) served as size markers. The lengths of some fragments are indicated. Protected major and minor fragments are indicated by arrows. (c) Suggested secondary structure of the $3^{\prime}$ portion of the scr gene, highlighting the secondary structure that may serve as the $\rho$-independent terminator. Arrows are according to (b).

region resembles a characteristic $E$. coli $\rho$-independent transcription terminator (Adhya \& Gottesman, 1978).

\section{Mapping the $5^{\prime}$ and $3^{\prime}$ ends of ScRNA}

To examine the invivo functionality of these transcriptional regulatory elements, both the $5^{\prime}$ and $3^{\prime}$ ends of scRNA were mapped. In addition, we investigated whether processing events occurred in B. brevis scRNA, since RNA processing is involved in the maturation of the B. subtilis scRNA. The $5^{\prime}$ end was identified by primer extension. Using the NK1 primer, only a 75 nucleotide band appeared (Fig. 3a), indicating that transcription starts from the $T$ residue at nucleotide position 473 (Fig. 2a). This position matched well with the $5^{\prime}$ end predicted by 
Table 1. Similarity and nucleotide distance data among scRNA gene sequences of Bacillus spp.

The upper right half of the table gives similarity values, $H$, for all pairwise comparisons of the scRNA sequences from 13 Bacillus species. $H$ is defined as in Sogin $e t$ al. (1986), where $H=m /(m+u+g / 2) ; m$ is the number of sequence positions with matching nucleotides in the two sequences, $u$ is the number of sequence with nonmatching nucleotides, and $g$ is the number of sequence positions that have a gap in one sequence opposite a nucleotide in the other. The absolute number of base changes and gapped positions is shown in the lower half of the table. The species names are abbreviated as follows: B. subtilis 168 (B. sub), B. amyloliquefaciens $\mathrm{H}(B$. amy), B. polymyxa ATCC 842 (B. pol), B. sphaericus 1593 (B. sph), B. pumilus BP1 (B. pum), B. macerans BKM B-51 (B. mac), B. megaterium 899 (B. meg), B. thuringiensis B4039 (B. thu), B. cereus T (B. cer), B. circulans A TCC 4513 (B. cir), B. stearotbermophilus 799 (B. ste), alkalophilic Bacillus C-125 (B. C125) and B. brevis ATCC 5246 (B. bre).

\begin{tabular}{|c|c|c|c|c|c|c|c|c|c|c|c|c|c|}
\hline & B. $s u b$ & B. $a m y$ & B. pol & B. $s p h$ & B. pum & B. $m a c$ & B. meg & B. $t h u$ & B. cer & B. cir & B. ste & B. C125 & B. bre \\
\hline B. sub & - & $1 \cdot 0$ & 0.998 & 0.985 & 0.969 & 0.958 & 0.895 & $0 \cdot 875$ & 0.879 & 0.877 & 0.868 & $0 \cdot 850$ & 0.732 \\
\hline B. $a m y$ & 0 & - & 0.998 & 0.985 & $0 \cdot 969$ & 0.958 & 0.895 & 0.875 & 0.879 & 0.877 & $0 \cdot 868$ & $0 \cdot 850$ & 0.732 \\
\hline B. pol & 1 & 1 & - & 0.983 & $0 \cdot 967$ & $0 \cdot 960$ & 0.889 & $0 \cdot 873$ & $0 \cdot 881$ & 0.879 & 0.862 & $0 \cdot 848$ & 0.729 \\
\hline B. $s p h$ & 1 & 4 & 5 & - & $0 \cdot 954$ & $0 \cdot 947$ & $0 \cdot 880$ & $0 \cdot 863$ & 0.875 & 0.866 & 0.852 & $0 \cdot 842$ & 0.721 \\
\hline B. pum & 9 & 9 & 10 & 13 & - & $0 \cdot 952$ & $0 \cdot 885$ & 0.869 & 0.877 & $0 \cdot 882$ & 0.869 & $0 \cdot 836$ & 0.705 \\
\hline B. mac & 12 & 12 & 11 & 15 & 14 & - & $0 \cdot 856$ & $0 \cdot 866$ & $0 \cdot 870$ & $0 \cdot 871$ & $0 \cdot 851$ & $0 \cdot 837$ & 0.716 \\
\hline B. $m e g$ & 29 & 29 & 31 & 33 & 31 & 40 & - & $0 \cdot 850$ & 0.866 & $0 \cdot 847$ & $0 \cdot 865$ & $0 \cdot 862$ & 0.695 \\
\hline B. $t b u$ & 35 & 35 & 36 & 38 & 36 & 38 & 41 & - & 0.978 & 0.839 & 0.852 & $0 \cdot 842$ & 0.711 \\
\hline B. cer & 36 & 36 & 35 & 37 & 36 & 38 & 39 & 9 & - & 0.844 & 0.853 & $0 \cdot 848$ & 0.727 \\
\hline B. cir & 34 & 34 & 33 & 37 & 33 & 35 & 43 & 44 & 45 & - & $0 \cdot 847$ & $0 \cdot 822$ & 0.728 \\
\hline B. ste & 37 & 37 & 38 & 40 & 36 & 41 & 37 & 41 & 43 & 42 & - & $0 \cdot 801$ & $0 \cdot 708$ \\
\hline B. C125 & 43 & 43 & 44 & 45 & 45 & $4 ?$ & 39 & 44 & 42 & 51 & 55 & - & $0 \cdot 705$ \\
\hline B. bre & 74 & 74 & 74 & 77 & 81 & 78 & 84 & 80 & 75 & 76 & 78 & 80 & - \\
\hline
\end{tabular}

alignment with the $B$. subtilis mature scRNA sequence (Fig. 2b). No other minor pre-scRNA was detected by primer extension.

For $3^{\prime}$ end mapping, a $0.6 \mathrm{kp} \mathrm{BamHI-EcoRI} \mathrm{fragment}$ encompassing the $3^{\prime}$ portion and the $3^{\prime}$ flanking region of the $B$. brevis scRNA gene (Fig. 1) was cloned into the riboprobe plasmid pBluescript II $\mathrm{SK}(-)$ and used to generate a ${ }^{32} \mathrm{P}$-labelled antisense RNA probe. RNA was prepared 3, 6, 9 and $12 \mathrm{~h}$ after inoculation. Fig. 3(b) shows three major and several minor protected RNA species. The three major species comprised 210, 206 and 204 nucleotides, among which the longest (210 nucleotides) may represent the $3^{\prime}$ end of mature scRNA. The $3^{\prime}$ end heterogeneity is probably an artifact caused during RNA preparation or the RNase protection assay, since the smaller protected RNA fragments varied in length with the experiment and RNA preparation. This suggests that the transcription of the scRNA gene terminates at the A residue at nucleotide position 745 . The $5^{\prime}$ and $3^{\prime}$ mapping study shows that the mature form of B. brevis scRNA consists of 273 nucleotides.

\section{Phylogeny of Bacillus scRNA}

Including the nucleotide sequence of $B$. brevis scRNA reported here, sequence data of scRNAs from 13 Bacillus species are now available. Gram-positive, rod-shaped, aerobic or facultatively anaerobic spore-forming bacteria have traditionally been assigned to the genus Bacillus. This genus as constituted in Bergey's Manual of Systematic Bacteriology (Claus \& Berkeley, 1986) is phenotypically heterogeneous, demonstrating an extremely wide range of nutritional requirements, growth conditions, metabolic diversity and DNA composition. A phylogenetic matrix study of the scRNA sequence also shows that the genus Bacillus is phylogenetically very heterogeneous (Table 1). $B$. brevis scRNA exhibited remarkably low sequence homologies with the other Bacillus groups. Based upon a comparison of the sequences of small-subunit-ribosomal RNA, Ash et al. (1991) have reported that the genus Bacillus can be separated into phylogenetically distinct groups. Among these, a group consisting of $B$. brevis and $B$. laterosporus forms a distinct line of descent exhibiting low levels of relatedness to all other Bacillus groups. This phylogenetic relationship agreed well with that deduced from scRNA. In contrast, a pairwise comparison of smallsubunit-ribosomal RNA reveals the very wide percentage sequence homology variation (range approximately 88$99 \%$ ). Therefore, the overall rate of divergence for scRNA is greater than that for $16 \mathrm{~S}$ rRNA.

Despite the relatively low sequence homology of the scRNA sequence between B. brevis and other Bacillus species, almost all nucleotide substitutions in scRNA are covariant and do not affect the secondary structure. That is, mutations that individually affect base pairing are often compensated by base changes on the other strand. Consequently, a strikingly similar secondary structure can be drawn for B. brevis scRNA based on that of B. subtilis (Struck \& Erdmann, 1990). The presence of a terminal loop, corresponding to domains I and II of human SRP RNA, is restricted to spore-forming Bacillus species among eubacteria. In domain I of B. subtilis scRNA, 
(a)

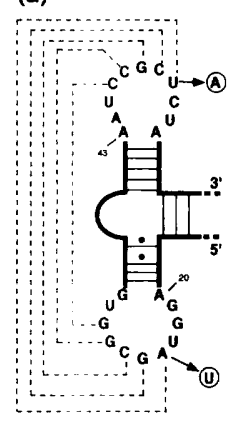

(b)

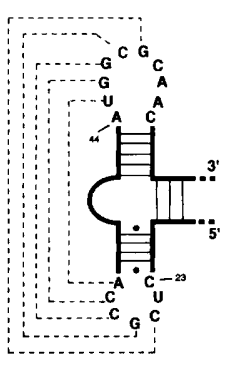

(c)



Fig. 4. Schematic drawing of two stem-loops in the domain I region of $B$. subtilis scRNA (a), Sulfolobus solfataricus 7S RNA (b), and human SRP RNA (c). Dashed lines show the possible tertiary interaction in each RNA. Circles denote the nucleotide changes in the $B$. brevis scRNA sequence, compared with that of B. subtilis. U.G and A.G base pairs are indicated by dots.

nucleotides 24-28 (5' AGCGG $3^{\prime}$ ) can pair with nucleotides 46-50 ( $\left.5^{\prime} \mathrm{CCGCU} 3^{\prime}\right)$. A potential tertiary interaction has been detected within this region (Fig. 4a). This interaction can be maintained in B. brevis scRNA by complementary base changes at nucleotide positions 24 and 50 (Fig. 4a). Therefore, this interaction is considered to also be important for the function of scRNA. It is notable that the same pairing can form in human SRP RNA and archaeobacterial 7S RNA (Fig. 4b, c), but not in yeast homologues.

On the other hand, the 22-nucleotide sequence in domain IV is completely identical among all Bacillus scRNAs. In eukaryotic SRP RNA and E. coli $4.5 \mathrm{~S}$ RNA, this conserved structure serves as the binding site for SRP54/ P48 proteins and has a pivotal role in RNA function in vivo (Selinger et al., 1993; Wood et al., 1993). Recently, we have cloned the $B$. subtilis gene encoding a homologue of SRP54/P48 (Honda et al., 1993). These results suggest that the corresponding region of scRNA of $B$. subtilis species functions in the same manner as eukaryotic and E. coli RNAs. Biochemical study and determination of the effects caused by mutations in domain IV may help in understanding of the function of this region.

\section{ACKNOWLEDGEMENTS}

Bacillus brevis ATCC 5246 was obtained from the Stock Center of Bacilli (Ohio State University). We thank Ms N. Foster for critical reading of the manuscript. This work was supported in part by Grants-in-Aid for Scientific Research from the Ministry of Education, Science and Culture of Japan.

\section{REFERENCES}

Adhya, S. \& Gottesman, M. (1978). Control of transcription. Annu Rev Biochem 47, 967-996.

Ash, C., Farrow, J. A. E., Wallbanks, S. \& Collins, M. D. (1991). Phylogenetic heterogeneity of the genus Bacillus revealed by comparative analysis of small-subunit-ribosomal RNA sequences. Lett Appl Microbiol 13, 202-206.
Claus, D. \& Berkeley, R. C. W. (1986). The genus Bacillus. In Bergey's Manual of Systematic Bacteriology, vol. 2, pp. 1105-1139. Edited by P. H. A. Sneath and others. Baltimore: Williams \& Wilkins.

Haas, E. S., Morse, D. P., Brown, J. W., Schmidt, F. J. \& Pace, N. R. (1991). Long range structure in ribonuclease P RNA. Science 254, 853-856.

Honda, K., Nakamura, K., Nishiguchi, M. \& Yamane, K. (1993). Cloning and characterization of a Bacillus subtilis gene encoding a homolog of the 54-kilodalton subunit of mammalian signal recognition particle and Eschericbia coli Ffh. $J$ Bacteriol 175, 4885-4894.

Kaine, B. P. (1990). Structure of the archaebacterial 7S RNA molecule. Mol \& Gen Genet 221, 315-321.

Larsen, N. \& Zwieb, C. (1991). SRP-RNA sequence alignment and secondary structure. Nucleic Acids Res 19, 17-24.

Lederberg, E. M. \& Cohen, S. N. (1974). Transformation of Salmonella typhimurium by plasmid deoxyribonucleic acid. J Bacteriol 119, 1072-1074.

Nakamura, K., Nakamura, A., Takamatsu, H. \& Yamane, K. (1990). Cloning and characterization of a Bacillus subtilis gene homologous to Escherichia coli secY.J Biochem 107, 603-607.

Nakamura, K., Imai, Y., Nakamura, A. \& Yamane, K. (1992a). Small cytoplasmic RNA of Bacillus subtilis: functional relationship with human signal recognition particle $7 \mathrm{~S}$ RNA and Escherichia coli $4.5 \mathrm{~S}$ RNA. J Bacteriol 174, 2185-2192.

Nakamura, K., Minemura, M., Nishiguchi, M., Honda, K. \& Yamane, K. (1992b). Conserved residues and secondary structure found in small cytoplasmic RNAs from Bacillus species. Nucleic Acids Res 20, 5227-5228.

Poritz, M., Strub, K. \& Walter, P. (1988). Human SRP RNA and Escherichia coli $4 \cdot 5 \mathrm{~S}$ RNA contain a highly homologous structural domain. Cell 55, 4-6.

Romero, D. P. \& Blackburn, E. (1991). A conserved secondary structure for telomerase RNA. Cell 67, 343-353.

Saito, H. \& Miura, K. (1963). Preparation of transforming deoxyribonucleic acid by phenol treatment. Biochim Biopbys Acta 72, 619-629.

Sanger, F., Nicklen, S. \& Coulson, A. R. (1977). DNA sequencing with chain terminating inhibitors. Proc Natl Acad Sci USA 74, 5463-5467.

Selinger, D., Brennwald, P., Liao, X. \& Wise, J. A. (1993). Identification of RNA sequence and structural elements required for assembly of fission yeast SRP54 protein with signal recognition particle RNA. Mol Cell Biol 13, 1353-1362.

Sogin, M. L., Ingold, A., Karlok, M., Nielsen, H. \& Engberg, J. (1986). Phylogenetic evidence for the acquisition of ribosomal RNA introns subsequent to the divergence of some of the major tetrahymena groups. EMBO J 5, 3625-3630.

Struck, J. C. R. \& Erdmann, V. A. (1990). Phylogenetic and biochemical evidence for a secondary structure model of a small cytoplasmic RNA from Bacilli. Eur J Biochem 192, 17-24.

Tinoco, I., Borer, P. N., Dengler, B. \& Levine, M. D. (1973). Improved estimation of secondary structure in ribonucleic acids. Nature New Biol 246, 40-41.

Walter, P. \& Blobel, G. (1980). Purification of a membrane associated protein complex required for protein translocation across the endoplasmic reticulum. Proc. Natl Acad Sci USA 77, 71127116.

Walter, P. \& Blobel, G. (1982). Signal recognition particle contains a 7S RNA essential for protein translocation across the endoplasmic reticulum. Nature 299, 691-698. 
Walter, P. \& Lingappa, V. (1986). Mechanism of protein translocation across the endoplasmic reticulum membrane. Annu Rei Cell Biol 2, 499-516.

Wood, H., Luirink, J. \& Tollervey, D. (1993). Evolutionarily' conserved nucleotides within the E. coli $4.5 \mathrm{~S}$ RNA are required for association with $\mathrm{P} 48$ in vitro and for optimal function in vivo. Nucleic Acids Res 20, 5919-5925.
Yanisch-Perron, C., Vieira, J. \& Messing, J. (1985). Improved M13 phage cloning vectors and host strains: nucleotide sequences of the M13mp18 and pUC19 vectors. Gene 33, 103-119.

Received 8 July 1993; revised 15 September 1993; accepted 21 September 1993. 